\title{
Teleportation of Superposition of Coherent States Using 4-Partite States and Effect of Decoherence on Fidelity
}

\author{
Shivani A. Kumar ${ }^{1 *}$, H. Prakash', N. Chandra², Ranjana Prakash ${ }^{2}$ \\ ${ }^{1}$ Amity Institute of Applied Sciences, Amity University, Noida, India \\ ${ }^{2}$ Department of Physics, University of Allahabad, Allahabad, India \\ Email: "'shivani_v11@rediffmail.com
}

Received May 22, 2012; revised July 12, 2012; accepted November 1, 2012

\begin{abstract}
A scheme of teleporting a superposition of coherent states $|\alpha\rangle$ and $|-\alpha\rangle$ using a 4-partite state, a beam splitter and two phase shifters was proposed by N. Ba An (Phys. Rev. A, 68, 022321, 2003). The author concluded that the probability for successful teleportation is only $1 / 4$ in the limit $|\alpha| \rightarrow \infty$ and $1 / 2$ in the limit $|\alpha| \rightarrow \infty$. In this paper it is shown that the author's scheme can be altered slightly so as to obtain an almost perfect teleportation for an appreciable value of $|\alpha|^{2}$. We find the minimum assured fidelity i.e., the minimum fidelity for an arbitrarily chosen information state, which we write MAF in this paper, for different cases. We also discuss the effect of decoherence on teleportation fidelity. We find that if no photons are counted in both final outputs, MAF, is still nonzero except when there is no decoherence and the initial state (the state to be teleported) is even coherent state. For non-zero photon counts, MAF decreases with increase in $|\alpha|^{2}$ for low noise. For high noise, however, it increases, attains a maximum value and then decreases with $|\alpha|^{2}$. The average fidelity depends appreciably on the initial state for low values of $|\alpha|^{2}$ only.
\end{abstract}

Keywords: Teleportation; Entanglement; Coherent States; Decoherence

\section{Introduction}

Quantum entanglement has generated much interest in many ingenious applications in quantum information science such as quantum teleportation [1-4], quantum computation [5], quantum dense coding [6], quantum cryptography [7] and quantum telecloning [8] as well as fundamental studies in quantum mechanics related to the Einstein-Podolsky-Rosen (EPR) paradox [9]. In a recent paper [10], a new idea of two-way quantum communication called "secure quantum information exchange" $(S Q I E)$ is also introduced. If there are two arbitrary unknown quantum states $|\xi\rangle I_{A}$ and $|\eta\rangle I_{B}$, initially with Alice and Bob, respectively, then SQIE protocol leads to the simultaneous exchange of these states between Alice and Bob with the aid of the special kind of six-qubit entangled (SSE) state and classical assistance of the third party, Charlie. Teleportation of a two-mode entangled coherent state encoded with two-qubit information has also been studied in a recent paper [11-15]. Teleportation has been demonstrated experimentally [14-16]. Teleportation of an entangled multiparticle state and coherent

"Corresponding author. superposition states have also been considered [17,18]. In addition to scheme for discrete variables, the idea was extended to continuous variables also both experimenttally [19] and theoretically [20-22]. Entangled coherent states $[23,24]$ have received much attention in the study of quantum entanglement and quantum teleportation. Effect of decoherence in teleportation has also been studied by various authors $[25,26]$. However, Fan and Lu An. [27] have used the terminology "coherent entangled states" which is completely different from "entangled coherent state". The entangled states [23] used by Sanders can be denoted as entangled coherent states in spite the crucial difference the phase factor makes. Recently teleportation of states such as

$$
|\psi\rangle \propto \epsilon_{+}|\alpha\rangle+\epsilon_{-}|-\alpha\rangle
$$

or

$$
|\phi\rangle \propto \epsilon_{+}|\alpha\rangle_{1}|\alpha\rangle_{2}+\epsilon_{-}|-\alpha\rangle_{1}|-\alpha\rangle_{2}
$$

with $|\alpha\rangle$ a coherent state and $\epsilon_{ \pm}$unknown complex coefficients, have been investigated by van Enk and Hirota [2] and $X$. Wang respectively [3]. However both the publications involved teleportation between two parties 
only.

In a recent paper $\mathrm{N}$. $\mathrm{Ba} \mathrm{An}$ [4] proposed a scheme to teleport a single particle state of form (1.1) within a network consisting of four parties, a beam splitter and two phase shifters and concluded that the probability for successful teleportation is only $1 / 4$ in the limit $|\alpha| \rightarrow 0$ and $1 / 2$ in the limit $|\alpha| \rightarrow \infty$. We [28-34] reexamined scheme of An and showed that an almost perfect teleporttation can be obtained for an appreciable value of $|\alpha|^{2}$. Also, by including four parties, the security of the teleportation increases, as David cannot decipher the original information until and unless he gets the results from all Alice, Bob and Clair. In this paper we discuss effect of decoherence on fidelity in such teleportation. We organize our paper as follows. In Section 2 we outline our teleportation scheme in Figure 1, which is self-explanatory and consider the case of no noise. In Section 3 we consider the effect of decoherence due to noise and in Sec- tion 4 we give conclusions.

While considering the noisy case, we find that if no photons are counted in both final outputs, the minimum assured fidelity, which we shall write MAF in this paper, is nonzero except when there is no decoherence and the initial state is even coherent state. For non-zero photon counts in all cases, MAF decreases with increase in $|\alpha|^{2}$ for low noise. For high noise, however, the MAF increases, attains a maximum value and then decreases with $|\alpha|^{2}$. Various cases have been studied extensively and the results plotted.

\section{Teleportation Scheme and the Noiseless Case}

Let us deal here with a network consisting of four parties: Alice, Bob, Clair and David. Because of the network symmetry requirement, the four parties share a 4-partite entangled state of the form

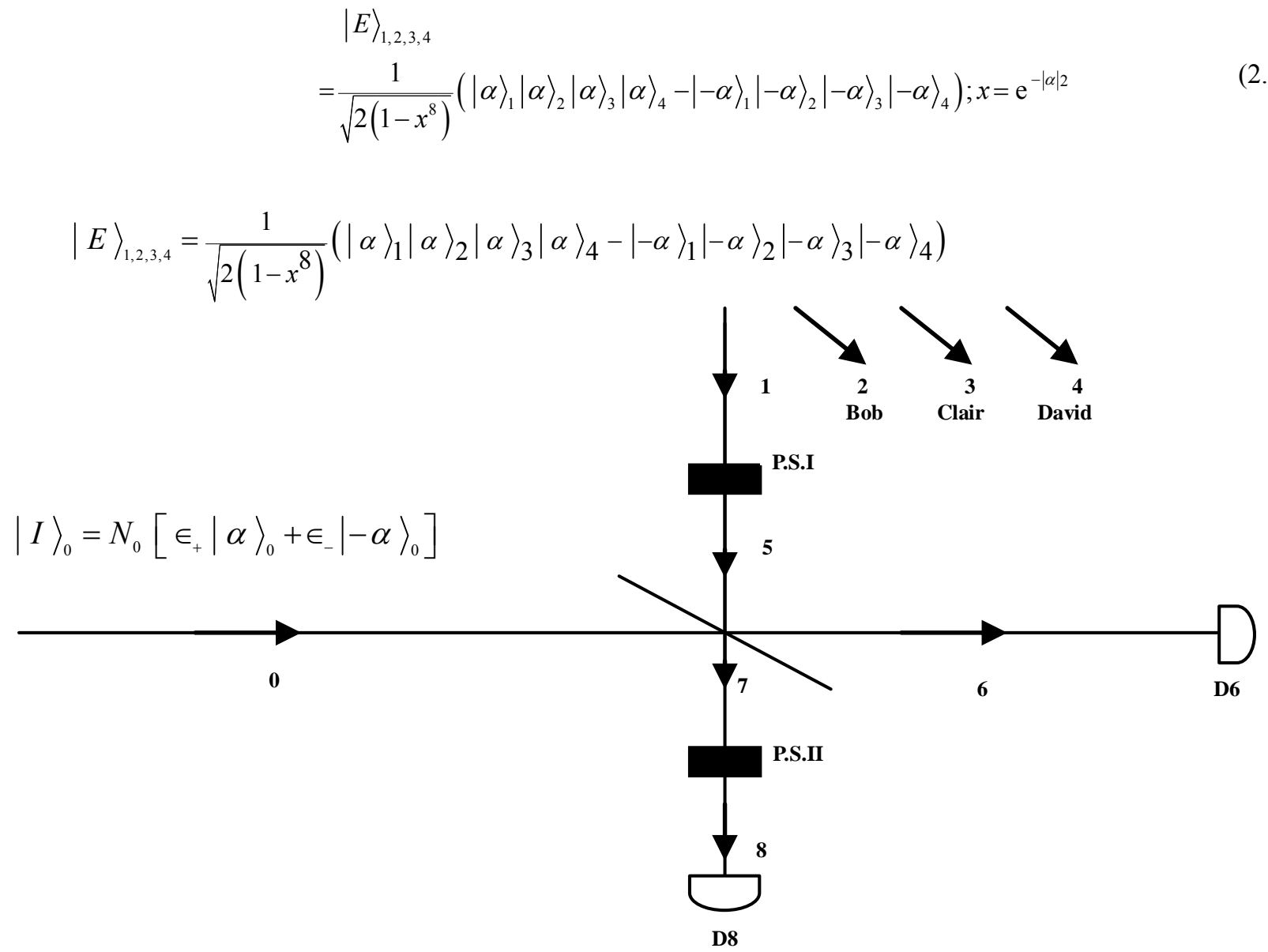

Figure 1. Numerals 0, 1, $\cdots, 8$ refers to modes. Out of $|\mathrm{E}\rangle_{1,2,3,4}, \mathbf{1 , 2 , 3}$ and 4 goes to Alice, Bob, Clair and David respectively.

Alice 1) converts state 1 to state 5 by using phase shifter P.S.I, 2) mixes state 5 with state 0 (information required to be teleported) using a beam splitter, 3) modifies output in 7 to state 8 using phase shifter P.S.II, and 4) performs photon counting in 6 and 8 . Bob and Clair also perform photon countings in modes 2 and 3 respectively. The result, conveyed to David by a classical channel helps him retrieve the information by making a suitable unitary transformation on state 4. 
involving modes $1,2,3$ and 4 . State with mode 1 is sent to Alice, mode 2 to Bob, mode 3 to Clair and mode 4 to David. Consider teleportation from Alice to David and let the state with Alice be,

$$
|I\rangle_{0}=\epsilon_{+}|\alpha\rangle_{0}+\epsilon_{-}|-\alpha\rangle_{0}
$$

in the mode 0 , with the normalization condition $\left|\epsilon_{+}\right|^{2}+\left|\epsilon_{-}\right|^{2}+2 \mathrm{e}^{-2|\alpha|^{2}} \operatorname{Re}\left(\epsilon_{+}^{*} \epsilon_{-}\right)=1$ We may call the state $|I\rangle_{0} \quad$ as the initial state. The mean number of photons is

$$
\bar{n}={ }_{0}\left\langle I\left|a^{+} a\right| I\right\rangle_{0}=|\alpha|^{2}\left[1-4 \mathrm{e}^{-2|\alpha|^{2}} \operatorname{Re}\left(\epsilon_{+}^{*} \epsilon_{-}\right)\right]
$$

which is nearly $|\alpha|^{2}$ for $|\alpha|^{2} \quad 1$. In terms of even and odd coherent states [35], the state $|I\rangle_{0}$ can be written as

$$
|I\rangle_{0}=A_{+}|E V E N, \alpha\rangle_{0}+A_{-}|O D D, \alpha\rangle_{0}=\cos \frac{\theta}{2}|E V E N, \alpha\rangle_{0}+\sin \frac{\theta}{2} \mathrm{e}^{\mathrm{i} \phi}|O D D, \alpha\rangle_{0}
$$

where

$$
|E V E N, \alpha\rangle=\frac{|\alpha\rangle+|-\alpha\rangle}{\sqrt{2\left(1+x^{2}\right)}},|O D D, \alpha\rangle=\frac{|\alpha\rangle-|-\alpha\rangle}{\sqrt{2\left(1-x^{2}\right)}}
$$

Constants $A_{ \pm}$and $\in_{ \pm}$are related to each other by,

$$
A_{ \pm}=\left(\epsilon_{+} \pm \epsilon_{-}\right) \sqrt{\left(1 \pm x^{2}\right) / 2}, \in_{ \pm}=\left[2\left(1 \pm x^{2}\right)\right]^{-1 / 2} A_{+} \pm\left[2\left(1-x^{2}\right)\right]^{-1 / 2} A_{-}
$$

and $\theta$ and $\phi$ are defined by,

$$
\tan \frac{\theta}{2} \mathrm{e}^{\mathrm{i} \phi}=A_{-} / A_{+}
$$

The initial state of the whole system is then given by, $|\Psi\rangle_{0,1,2,3,4}=|I\rangle_{0}|E\rangle_{1,2,3,4}$. Alice allows mode 1 to pass through phase shifter P.S.I, which converts state $|\alpha\rangle_{1}$ in mode 1 to state $|-i \alpha\rangle_{5}$ in mode 5 . She then mixes modes 0 and 5 with the help of a beam splitter which changes [36] an input state $|\beta\rangle_{0}|\gamma\rangle_{5}$ to the state

$$
\left|\frac{1}{\sqrt{2}}(\beta+i \gamma)\right\rangle_{6}\left|\frac{1}{\sqrt{2}}(\gamma+i \beta)\right\rangle_{7}
$$

in modes 6 and 7. The state after passing through the beam splitter is then,

$$
\begin{aligned}
& |\Psi\rangle_{6,7,2,3,4} \\
& =\frac{1}{\sqrt{2\left(1-x^{8}\right)}}\left[\epsilon_{+}\left(|\sqrt{2} \alpha\rangle_{6}|0\rangle_{7}|\alpha\rangle_{2}|\alpha\rangle_{3}|\alpha\rangle_{4}-|0\rangle_{6}|i \sqrt{2} \alpha\rangle_{7}|-\alpha\rangle_{2}|-\alpha\rangle_{3}|-\alpha\rangle_{4}\right)\right. \\
& \left.\quad+\epsilon_{-}\left(|0\rangle_{6}|-i \sqrt{2} \alpha\rangle_{7}|\alpha\rangle_{2}|\alpha\rangle_{3}|\alpha\rangle_{4}-|-\sqrt{2} \alpha\rangle_{6}|0\rangle_{7}|-\alpha\rangle_{2}|-\alpha\rangle_{3}|-\alpha\rangle_{4}\right)\right]
\end{aligned}
$$

One of the output modes, say, mode 7 is allowed to pass through phase shifter P.S.II which changes a state $|\eta\rangle_{7}$ to the state $|i \eta\rangle_{8}$, the whole process changes the combined state $|\Psi\rangle_{6,7,2,3,4}$ to state

$$
\begin{aligned}
& |\Psi\rangle_{6,8,2,3,4} \\
& =\frac{1}{\sqrt{2\left(1-x^{8}\right)}}\left[\epsilon_{+}\left(|\sqrt{2} \alpha\rangle_{6}|0\rangle_{8}|\alpha\rangle_{2}|\alpha\rangle_{3}|\alpha\rangle_{4}-|0\rangle_{6}|-\sqrt{2} \alpha\rangle_{8}|-\alpha\rangle_{2}|-\alpha\rangle_{3}|-\alpha\rangle_{4}\right)\right. \\
& \left.\quad+\epsilon_{-}\left(|0\rangle_{6}|\sqrt{2} \alpha\rangle_{8}|\alpha\rangle_{2}|\alpha\rangle_{3}|\alpha\rangle_{4}-|-\sqrt{2} \alpha\rangle_{6}|0\rangle_{8}|-\alpha\rangle_{2}|-\alpha\rangle_{3}|-\alpha\rangle_{4}\right)\right]
\end{aligned}
$$

This scheme of teleportation is shown in Figure 1.

Using the expansion $| \pm \sqrt{2} \alpha\rangle$

[28-34,37],

$$
| \pm \sqrt{2} \alpha\rangle=x|0\rangle+\frac{\left(1-x^{2}\right)}{\sqrt{2}}|N Z E, \sqrt{2} \alpha\rangle \pm \sqrt{\frac{1}{2}\left(1-x^{4}\right)}|O D D, \sqrt{2} \alpha\rangle,
$$

where 


$$
|N Z E, \alpha\rangle=(|\alpha\rangle+|-\alpha\rangle-2 \sqrt{x}|0\rangle) / \sqrt{2}(1-x)
$$

The output state becomes

$$
\begin{aligned}
|\Psi\rangle_{6,8,2,3,4}= & \frac{x}{\sqrt{2\left(1-x^{8}\right)}}|0\rangle_{6}|0\rangle_{8}\left(\epsilon_{+} \epsilon_{-}\right)\left[|\{\alpha\}\rangle_{234}-|\{-\alpha\}\rangle_{234}\right] \\
& +\frac{1}{\sqrt{2\left(1+x^{2}\right)\left(1+x^{4}\right)}}\left\{|N Z E, \sqrt{2} \alpha\rangle_{6}|0\rangle_{8}\left[\epsilon_{+}|\{\alpha\}\rangle_{234}-\epsilon_{-}|\{-\alpha\}\rangle_{234}\right]\right. \\
& \left.+|0\rangle_{6}|N Z E, \sqrt{2} \alpha\rangle_{8}\left[-\epsilon_{+}|\{-\alpha\}\rangle_{234}+\epsilon_{-}|\{\alpha\}\rangle_{234}\right]\right\} \\
& +\frac{1}{\sqrt{2\left(1+x^{4}\right)}}\left\{|O D D, \sqrt{2} \alpha\rangle_{6}|0\rangle_{8}\left[\epsilon_{+}|\{\alpha\}\rangle_{234}+\epsilon_{-}|\{-\alpha\}\rangle_{234}\right]\right. \\
& \left.+|0\rangle_{6}|O D D, \sqrt{2} \alpha\rangle_{8}\left[\epsilon_{+}|\{-\alpha\}\rangle_{234}+\epsilon_{-}|\{\alpha\}\rangle_{234}\right]\right\}
\end{aligned}
$$

where,

$$
|\{\alpha\}\rangle_{234}=|\alpha\rangle_{2}|\alpha\rangle_{3}|\alpha\rangle_{4}
$$

Now, Alice performs a two-mode number measurement on the modes 6 and 8 through two detectors $D 6$ and $D 8$ at her station and conveys this result to David through some classical channel. Bob and Clair also carry out local photon number measurements of modes 2 and 3 by their detectors $D 2$ and $D 3$. After receiving this information from Alice, Bob and Clair, David makes some unitary transformation to retrieve the original information. Let the measurement outcomes of Alice be $n_{6}$ and $n_{8}$, whereas $n_{3}$ photons are counted by Bob and $n_{3}$ by Clair's detector.

From Equation (2.11), it is clear that Alice's result of photon countings of modes 6 and 8 is either 1) zero in both outputs, or 2) zero in mode 6 and non-zero even in the mode 8 , or 3 ) non-zero even in the mode 6 and zero in the mode 8 , or 4 ) zero in mode 6 and odd in the mode 8 or 5 ) odd in the mode 6 and zero in the mode 8 . We considered [24] different cases of results of photon counting in modes 6 and 8 . For $n_{2}$ and $n_{3}$, also, we distinguish in two cases, viz., (a) $n_{2}+n_{3}$ is even or (b) $n_{2}+$ $n_{3}$ is odd.

Case I: If Alice's measurements result in zero photons in both modes, we find that the state with David is the non entangled state,

$$
\left|T^{\prime}\right\rangle \quad\left(\epsilon_{+}+\epsilon_{-}\right)\left[|\{\alpha\}\rangle_{234}-|\{-\alpha\}\rangle_{224}\right]
$$

It may be noted that sign $\sim$ has been used in Equation (2.13) because normalization of the state has not been done. Also prime in $\left|T^{\prime}\right\rangle$ reminds that a unitary transformation $U$ is to be done by David resulting in teleported state $|T\rangle=U\left|T^{\prime}\right\rangle$. We may write the states $| \pm \alpha, \pm \alpha\rangle_{2,3}$ in terms of even and odd coherent states of modes 2 and 3 (see A1 in Appendix A).

For case $I$ (a), where, $n_{2}+n_{3}$ is even, David's state collapses into the state $|O D D, \alpha\rangle$ which is the odd coherent state. By applying unitary transformations, it can't be converted to state $|I\rangle$ for arbitrary values of $A_{ \pm}$. This gives maximum fidelity equal to 1 for information $|O D D, \alpha\rangle$ and fidelity zero for information $|E V E N, \alpha\rangle$. The MAF is thus zero in this case.

For case I (b), however, where $n_{2}+n_{3}$ is odd, David's state collapses into the state $|E V E N, \alpha\rangle$. Again, unitary transformation can't convert it to state $|I\rangle$ in this case and maximum fidelity is equal to 1 for information $|E V E N, \alpha\rangle$ and fidelity zero for information $|O D D, \alpha\rangle$. MAF is again zero in this case.

The probability of getting the state (2.13) is,

$$
P_{I}={ }_{23468}\langle\Psi \mid 0,0\rangle_{6868}\langle 0,0 \mid \Psi\rangle_{23468}=\frac{2 x^{2}\left(1-x^{6}\right)\left|A_{+}\right|^{2}}{\left(1-x^{8}\right)\left(1+x^{2}\right)}=\frac{2 x^{2}\left(1-x^{6}\right) \cos ^{2}\left(\frac{\theta}{2}\right)}{\left(1-x^{8}\right)\left(1+x^{2}\right)}
$$

where $A_{ \pm}$are related to $\theta$ by Equation (2.6). The probability $P_{I}$ approaches zero for an appreciable value of $|\alpha|^{2}$.
Case II: If Alice's measurements result in non-zero even photons in the mode 6 and zero photon in the mode 8 , we find that the state with David is, 


$$
\begin{aligned}
& \left|T^{\prime}\right\rangle \\
& {\left[\epsilon_{+}|\{\alpha\}\rangle_{234}-\epsilon_{-}|\{-\alpha\}\rangle_{234}\right]}
\end{aligned}
$$

The above state can be written in terms of even and odd coherent states of modes 2 and 3 (see A2 in Appen$\operatorname{dix}$ A).

For case II (a), where $\boldsymbol{n}_{2}+\boldsymbol{n}_{3}$ is even, David's state collapses into the state,

$$
\left|T^{\prime}\right\rangle \quad\left[\epsilon_{+}|\alpha\rangle_{4}-\epsilon_{-}|-\alpha\rangle_{4}\right]=A_{+} \sqrt{\frac{1-x^{2}}{1+x^{2}}}|O D D, \alpha\rangle_{4}+A_{-} \sqrt{\frac{1+x^{2}}{1-x^{2}}}|E V E N, \alpha\rangle_{4}
$$

Applying the unitary transformation

$$
U=|E V E N, \alpha\rangle_{4{ }_{4}}\langle O D D, \alpha|+| O D D, \alpha\rangle_{4}{ }_{4}\langle E V E N, \alpha|
$$

Equation (2.16) reduces to

$$
|T\rangle \quad A_{+} \sqrt{\frac{1-x^{2}}{1+x^{2}}}|E V E N, \alpha\rangle_{4}+A_{-} \sqrt{\frac{1+x^{2}}{1-x^{2}}}|O D D, \alpha\rangle_{4}
$$

and leads to the fidelity $F$ given by,

$$
F=\langle T \mid I\rangle\langle I \mid T\rangle=\frac{\left[1-x^{2}\left(\left|A_{+}\right|^{2}-\left|A_{-}\right|^{2}\right)\right]^{2}}{1+x^{4}-2 x^{2}\left(\left|A_{+}\right|^{2}-\left|A_{-}\right|^{2}\right)}=\frac{\left(1-x^{2} \cos \theta\right)^{2}}{1+x^{4}-2 x^{2} \cos \theta}
$$

This is minimum at $\theta=\cos ^{-1}\left(x^{2}\right)$. The MAF is the minimum value of $F, F_{\min }=\left(1-x^{4}\right)$. This is quite close to unity for appreciable value of $|\alpha|^{2}$.

For case II (b), where $n_{2}+n_{3}$ is odd, David's state collapses into the state,

$$
\begin{aligned}
& \left|T^{\prime}\right\rangle \sim\left[\epsilon_{+}|\alpha\rangle_{4}+\epsilon_{-}|-\alpha\rangle_{4}\right] \\
= & A_{+}|E V E N, \alpha\rangle_{4}+A_{-}|O D D, \alpha\rangle_{4}
\end{aligned}
$$

This is an exact replica of state $|I\rangle_{0}$, and hence no unitary transformation is required for this case. The fidelity is obviously unity.

Probability for getting the state $(2.15)$ is

$$
\begin{aligned}
P_{I I} & ={ }_{23468}\langle\Psi \mid N Z E, 0\rangle_{68}{ }_{68}\langle 0, N Z E \mid \Psi\rangle_{23468} \\
& =\frac{\left[1+x^{8}-x^{2}\left(1+x^{4}\right)\left(\left|A_{+}\right|^{2}-\left|A_{-}\right|^{2}\right)\right]}{4\left(1+x^{4}\right)\left(1+x^{2}\right)^{2}} \\
& =\frac{\left[1+x^{8}-x^{2}\left(1+x^{4}\right) \cos \theta\right]}{4\left(1+x^{4}\right)\left(1+x^{2}\right)^{2}}
\end{aligned}
$$

This is very close to $1 / 4$ for an appreciable value of $|\alpha|^{2}$.

$$
\left|T^{\prime}\right\rangle \sim\left[\epsilon_{+}|\alpha\rangle_{4}+\epsilon_{-}|-\alpha\rangle_{4}\right]=A_{+}|E V E N, \alpha\rangle_{4}-A_{-}|O D D, \alpha\rangle_{4}
$$

Applying the unitary transformation,

$$
U=|E V E N, \alpha\rangle_{44}\langle E V E N, \alpha|-| O D D, \alpha\rangle_{44}\langle O D D, \alpha|
$$

Equation (2.25) becomes an exact replica of state $|I\rangle_{0}$ and hence the fidelity comes out to be unity. The prob- ability of getting the state (2.22) is same as Equation (2.21). 
Case IV: If Alice's measurements result in odd photons in the mode 6 and zero photon in the mode 8 , we find that the state with David is,

$$
\left|T^{\prime}\right\rangle\left[\epsilon_{+}|\{\alpha\}\rangle_{234}+\epsilon_{-}|\{-\alpha\}\rangle_{234}\right]
$$

The above state can be written in terms of even and odd coherent states of modes 2 and 3 (see A4 in Appen$\operatorname{dix}$ A)

For case IV (a), where $n_{2}+n_{3}$ is even, David's state collapses into the state,

$$
\begin{aligned}
& \left|T^{\prime}\right\rangle \sim\left[\epsilon_{+}|\alpha\rangle_{4}+\epsilon_{-}|-\alpha\rangle_{4}\right] \\
= & A_{+}|E V E N, \alpha\rangle_{4}+A_{-}|O D D, \alpha\rangle_{4}
\end{aligned}
$$

This is an exact replica of state $|I\rangle_{0}$ and hence no unitary transformation is required for this case and the fidelity is unity.

For case IV (b) where $\boldsymbol{n}_{2}+\boldsymbol{n}_{3}$ is odd, David's state collapses into the state,

$$
\left.\left|T^{\prime}\right\rangle \sim\left[\epsilon_{+}|\alpha\rangle_{4}-\epsilon_{-}|-\alpha\rangle_{4}\right]=A_{+} \sqrt{\frac{1-x^{2}}{1+x^{2}}}|O D D, \alpha\rangle_{4}+A_{-} \sqrt{\frac{1+x^{2}}{1-x^{2}}} \mid \text { EVEN, } \alpha\right\rangle_{4}
$$

This is exactly the same as case II (a).

Probability of getting the state (2.27) is

$$
P_{I V}={ }_{23468}\langle\Psi \mid O D D, 0\rangle_{68}{ }_{68}\langle 0, O D D \mid \Psi\rangle_{23468}=\frac{\left[1+x^{4}-x^{2}\left(\left|A_{+}\right|^{2}-\left|A_{-}\right|^{2}\right)\right]}{4\left(1+x^{4}\right)}=\frac{\left[1+x^{4}-x^{2} \cos \theta\right]}{4\left(1+x^{4}\right)}
$$

This is very close to $1 / 4$ for an appreciable value of $|\alpha|^{2}$.

Case V: If Alice's measurements result in zero photon in the mode 6 and odd photon in the mode 8 , we find that the state with David is,

$$
\left|T^{\prime}\right\rangle \sim\left[\epsilon_{+}|\{-\alpha\}\rangle_{234}+\epsilon_{-}|\{\alpha\}\rangle_{234}\right]
$$

In terms of even and odd coherent states of modes 2 and 3 , the above state becomes,
For case $\mathbf{V}(\mathrm{a})$, where $\boldsymbol{n}_{2}+\boldsymbol{n}_{3}$ is even, David's state collapses into the state,

$$
\begin{aligned}
& \left|T^{\prime}\right\rangle \sim\left[\epsilon_{+}|-\alpha\rangle_{4}+\epsilon_{-}|\alpha\rangle_{4}\right] \\
= & A_{+}|E V E N, \alpha\rangle_{4}-A_{-}|O D D, \alpha\rangle_{4}
\end{aligned}
$$

This is exactly the same as case III (b).

For case V (b), where $n_{2}+n_{3}$ is odd, David's state collapses into the state,

$$
\left|T^{\prime}\right\rangle \sim\left[-\epsilon_{+}|-\alpha\rangle_{4}+\epsilon_{-}|\alpha\rangle_{4}\right]=A_{+} \sqrt{\frac{1-x^{2}}{1+x^{2}}}|O D D, \alpha\rangle_{4}-A_{-} \sqrt{\frac{1+x^{2}}{1-x^{2}}}|E V E N, \alpha\rangle_{4}
$$

This is exactly the same as case III (a). The average fidelity i.e., $F_{a v}=\sum_{i=I} P_{i} F_{i}$ is seen to have
the minimum value

$$
F_{a v, \min }=1-2\left(1+x^{2}\right)^{-2} x^{2} \text { for } A_{-}=0 \text {. For }|\alpha|^{2}=5
$$

the value of $F_{a v, \min }$ is 0.9999 , which is very close to unity and we lead to the conclusion that the teleportation is almost successful.

\section{Effect of Noise on Teleportation}

Following van Enk and Hirota [2-28] we assume that the initial state and the entangled state suffer losses, i.e., the modes $0,1,2,3$ and 4 are attenuated as they travel through a noisy channel and photons are transferred to reservoir modes making each of modes $0,1,2,3$ and 4 to have transformations of the type,

$$
|\alpha\rangle|0\rangle_{R} \rightarrow|\sqrt{\eta} \alpha\rangle|\sqrt{1-\eta} \alpha\rangle_{R}
$$

where the second state $|0\rangle_{R}$ refers to the part of "reservoir" which interacts with the state $|\alpha\rangle$ in question and $\eta$ is the noise parameter, which gives the fraction of photons that survive the noise. The part of reservoir coupled to mode $0,1,2,3$ and 4 will be denoted by $R 0, R 1, R 2, R 3$ and $R 4$ respectively. In presence of noise, the state to be teleported (Equation (2.2)) develops into the state,

$$
|I\rangle_{0}|0\rangle_{R 0} \rightarrow|I\rangle_{0, R 0}=\epsilon_{+}|\alpha\rangle_{0}|k\rangle_{R 0}+\epsilon_{-}|-\alpha\rangle_{0}|-k\rangle_{R 0} ; \alpha=\sqrt{\eta} \alpha, k=\sqrt{1-\eta} \alpha
$$

Here $R 0$ refers to the part of reservoir coupled to mode 0 having the information state $|I\rangle_{0}$. If $R 0$ con- tains more than one reservoir modes coupled to state $|0\rangle$ then, $|k\rangle_{R 0}$ stands for 


$$
\begin{gathered}
\prod_{i}\left|k_{i}\right\rangle_{R 0 i},|k|^{2}=\sum_{i}\left|k_{i}\right|^{2}=(1-\eta)|\alpha|^{2} . \\
|E\rangle_{1,2,3,4}=\frac{1}{\sqrt{2\left(1-x^{8}\right)}}\left(|\alpha\rangle_{1}|\alpha\rangle_{2}|\alpha\rangle_{3}|\alpha\rangle_{4}|K\rangle_{R 1234}-|-\alpha\rangle_{1}|-\alpha\rangle_{2}|-\alpha\rangle_{3}|-\alpha\rangle_{4}|-K\rangle_{R 1234}\right)
\end{gathered}
$$

where

$$
|K\rangle_{R 1234}=|k\rangle_{R 1}|k\rangle_{R 2}|k\rangle_{R 3}|k\rangle_{R 4}=|\sqrt{1-\eta} \alpha\rangle_{R 1}|\sqrt{1-\eta} \alpha\rangle_{R 2}|\sqrt{1-\eta} \alpha\rangle_{R 1}|\sqrt{1-\eta} \alpha\rangle_{R 1}
$$

or

$$
|K\rangle_{R 12}=\prod_{i}\left|k_{1 i}\right\rangle_{R 1 i}\left|k_{2 i}\right\rangle_{R 2 i}, \sum_{i}\left|k_{1 i}\right|^{2}=\sum\left|k_{2 i}\right|^{2}=(1-\eta)|\alpha|^{2}
$$

We assume that the reservoir modes remain unaffected by phase shifter and the beam splitter and following the same steps as in Section 2, the state $|\Psi\rangle_{4,6,8, R 0, R 1234}$ is
seen to be

$$
\begin{aligned}
|\Psi\rangle_{4,6,8, R 0, R 1234} & \frac{x}{\sqrt{2\left(1-x^{4}\right)}}|0\rangle_{6}|0\rangle_{8}\left[\left(\epsilon_{+}+\epsilon_{-}\right)\left(|\{\alpha\}\rangle_{234}|r\rangle-|[-\alpha]\rangle_{234}\left|-r^{\prime}\right\rangle\right)\right] \\
& +\frac{1-x^{2}}{2 \sqrt{1-x^{4}}}\left\{|N Z E, \sqrt{2} \alpha\rangle_{6}|0\rangle_{8}\left[\epsilon_{+}|\{\alpha\}\rangle_{234}|r\rangle-\epsilon_{-}|-\{\alpha\}\rangle_{234}|-r\rangle\right]\right. \\
& \left.+|0\rangle_{6}|N Z E, \sqrt{2} \alpha\rangle_{8}\left[-\epsilon_{+}|\{-\alpha\}\rangle_{234}\left|-r^{\prime}\right\rangle+\epsilon_{-}|\{\alpha\}\rangle_{234}\left|-r^{\prime}\right\rangle\right]\right\} \\
& +\frac{1}{2} \sqrt{\frac{1-x^{4}}{1-x^{4}}}\left\{|O D D, \sqrt{2} \alpha\rangle_{6}|0\rangle_{8}\left[\epsilon_{+}|\{\alpha\}\rangle_{234}|r\rangle+\epsilon_{-}|\{-\alpha\}\rangle_{234}|-r\rangle\right]\right. \\
& \left.+|0\rangle_{4}|O D D, \sqrt{2} \alpha\rangle_{6}\left[\epsilon_{+}|\{-\alpha\}\rangle_{234}\left|-r^{\prime}\right\rangle+\epsilon_{-}\left|\{\alpha\}^{\prime}\right\rangle_{234}\left|r^{\prime}\right\rangle\right]\right\}
\end{aligned}
$$

where

$$
|\{ \pm \alpha\}\rangle_{234}=| \pm \alpha\rangle_{2}| \pm \alpha\rangle_{3}| \pm \alpha\rangle_{4}, x \equiv \mathrm{e}^{-|\alpha|^{2}},|r\rangle \equiv\left|k_{0}\right\rangle_{R 0}|K\rangle_{R 1234} \text { and }\left|r^{\prime}\right\rangle \equiv\left|-k_{0}\right\rangle_{R 0}|K\rangle_{R 1234}
$$

Tilde in $|\Psi\rangle$ or anywhere else reminds that decoherence because of noise is being accounted for. It should be noted that the result of photon counting in modes 6 and 8 is always zero in one of the two modes and 1) zero or, 2) non-zero even or 3) odd in the other mode. In Section 2, we concluded that 1) fidelity is unity when total photon counts $n_{6}+n_{8}+n_{2}+n_{3}$ are odd. In this case further, if $n_{8}=0$, no unitary transformation is required;2) if $n_{6}=0$, this requires a unitary transformations which changes the states $| \pm \alpha\rangle$ to $|\alpha\rangle$; and 3) for other results no unitary transformations exist which may convert perfectly the state in mode 2 to the information state. This was also the reason behind conclusion of $\mathrm{N}$. $\mathrm{Ba}$ An that the teleportation is not possible when the result of photon counting is even. Here also, we apply the same unitary transformations as in Section 2 above with a change that $\alpha$ is now replaced by $\alpha$. For discussion of different cases the information coefficients $\epsilon_{ \pm}$in (3.7) will be expressed in terms of $A_{ \pm}$by using (2.4). Also, as discussed earlier, for obtaining fidelity we use the initial information $|I\rangle$ and not the decohered information $|I\rangle$, as our aim is to teleport the information $|I\rangle$. (3.7) may be compared to (2.13) and it may be seen that the state $|\Psi\rangle_{6,8,2,3,4}$ is a 5-mode state which reduces to the teleported state $|T\rangle_{4}$ with Bob after photon counting measurements in modes 6 and 8 . The state $|\Psi\rangle$, however contains five reservoir modes also and hence after photon counting measurements it leads to state $|T\rangle$ of modes 2-4, $R 0$ and $R 1234$, which contains the teleported state with Bob entangled with the reservoir states. The reduced state with Bob can be expressed by a reduced density operator $\rho_{B o b}=\operatorname{Tr}_{R 0, R 12} \rho_{T}$ where $\rho_{T}=|T\rangle\langle T|$. The fidelity of teleportation should obvi-
ously be 


$$
F=\langle T \mid I\rangle_{4}{ }_{4}\langle I \mid T\rangle=\operatorname{Tr}\left[\rho_{I} \rho_{T}\right]
$$

where $|I\rangle_{4}$ is the considered state in mode 4 identical with the state $|I\rangle_{0}$. We can now discuss the various results of photon counting. We shall report the MAF for each case so that minimum success of teleportation is evident.

Case I: If Alice's measurements result in zero photons in both modes, we find that the state with David is the non entangled state,

$$
\left|T^{\prime}\right\rangle \sim\left(\epsilon_{+}|k\rangle_{R 0}+\epsilon_{-}|-k\rangle_{R 0}\right)\left[|\{\alpha\}\rangle_{234}|K\rangle_{R 1234}-|\{-\alpha\}\rangle_{234}|-K\rangle_{R 1234}\right]
$$

For case I (a), where, $n_{2}+n_{3}$ is even, the fidelity $F_{I E}$ (see B5 in Appendix B) can easily be found by (3.9). Although no unitary transformation for successful teleportation is possible, we still have a nonzero fidelity in this case. Putting

$$
\begin{gathered}
\left|A_{+}\right|=\cos \frac{\theta}{2} \text { and }\left|A_{-}\right|=\sin \frac{\theta}{2} \quad \begin{array}{r}
\cosh 2 \chi<\left(1+x^{2} p^{8}\right) /\left(x^{2}+p^{8}\right) \\
\text { These minimum values are respectively, }
\end{array} \\
F_{\min }=\frac{2 x^{2}\left(1-p^{8}\right) \cosh ^{2} \chi}{p\left(1+x^{2}\right)\left(1-x^{2} p^{6}\right)} \text { at } \theta=0, F_{\min }=\frac{2 x^{2}\left(1+p^{8}\right) \sinh ^{2} \chi}{p\left(1-x^{2}\right)\left(1-x^{2} p^{6}\right)} \text { at } \theta=\pi
\end{gathered}
$$

where

$$
p=\exp \left[-(1-\eta)|\alpha|^{2}\right], \chi=|\alpha|^{2} \sqrt{\eta}
$$

We note that, unlike the previous noiseless case where MAF was zero, MAF is nonzero here in general. It is zero only when there is no decoherence and the initial we find that the expression (B4) of fidelity has one minimum. The minimum is at $\theta=0$ if

or at $\theta=\pi$ if

$$
\cosh 2 \chi>\left(1+x^{2} p^{8}\right) /\left(x^{2}+p^{8}\right)
$$

state is an even coherent state.

For case $I(b)$, where, $\boldsymbol{n}_{\mathbf{2}}+\boldsymbol{n}_{\mathbf{3}}$ is odd, the fidelity $F_{I O}$ (see B10 in Appendix B) again has one minimum. The minimum is at $\theta=0$ if $\cosh 2 \chi>\left(1-x^{2} p^{8}\right) /\left(x^{2}-p^{8}\right)$, or at $\theta=\pi$ if $\cosh 2 \chi<\left(1-x^{2} p^{8}\right) /\left(x^{2}-p^{8}\right)$. These minimum values are respectively,

$$
F_{\min }=\frac{2 x^{2}\left(1+p^{8}\right) \cosh ^{2} \chi}{p\left(1+x^{2}\right)\left(1+x^{2} p^{6}\right)} \text { at } \theta=0, F_{\min }=\frac{2 x^{2}\left(1-p^{8}\right) \sinh ^{2} \chi}{p\left(1-x^{2}\right)\left(1+x^{2} p^{6}\right)} \text { at } \theta=\pi
$$

Case II: If Alice's measurements result in non-zero even photons in the mode 6 and zero photon in the mode 8 , we find that the state with David is,

$$
\left|T^{\prime}\right\rangle \sim\left[\epsilon_{+}|\{\alpha\}\rangle_{234}|r\rangle-\epsilon_{-}|\{-\alpha\}\rangle_{234}|-r\rangle\right]
$$

For case II (a), where $n_{2}+n_{3}$ is even, David may apply the same unitary transformation as in Section 2 above, with the only change that $\alpha$ is now replaced by $\alpha$. The fidelity $F_{I I E}$ (see (B17) in Appendix B) can again be found by (3.9).

We may find the value of MAF by putting

$$
\left|A_{+}\right|=\cos \frac{\theta}{2} \text { and }\left|A_{-}\right|=\sin \frac{\theta}{2}
$$

and choosing the phase factor $\phi$ between $A_{+}$and $A_{-}$, $\phi=\pi / 2$ so as to make $\left(A_{+}^{2} A_{-}^{* 2}+A_{-}^{2} A_{+}^{* 2}\right)$ minimum. Figure 2 shows variation of MAF with $|\alpha|^{2}$ for different values of $\eta$. It is again seen that, for the noisy case MAF increases, attains a maximum value and then decreases with $|\alpha|^{2}$. This maximum value decreases as $|\alpha|^{2}$ increases.

For case II (b), where $n_{2}+n_{3}$ is odd, no unitary transformation is required. The fidelity $F_{I I O}$ is given by the expression (B22) in Appendix B. We can find the MAF by adjusting the phase factor $\phi$ between $A_{+}$and $A_{-}$and by varying $\theta$.

Variation of MAF with $|\alpha|^{2}$ for different values of $\eta$ is shown in Figure 3. For the noisy case, it is seen that MAF decreases uniformly with increase in $|\alpha|^{2}$ for small noise. However on increasing noise, a maximum appears at $|\alpha|^{2}=0$ followed by another maximum and the maximum value decreases on increasing noise.

Case III: If Alice's measurements result in zero photon in the mode 6 and non-zero even photons in the mode 8 , we find that the state with David is,

$$
\left|T^{\prime}\right\rangle \sim\left[-\epsilon_{+}|\{-\alpha\}\rangle_{234}\left|-r^{\prime}\right\rangle+\epsilon_{-}|\{\alpha\}\rangle_{234}\left|r^{\prime}\right\rangle\right]
$$

For case III (a), where $n_{2}+n_{3}$ is even, after the required unitary transformation (see B26), this lead to $F_{I I I E}=F_{I I E}$. Discussions of the previous case are valid here also.

For case III (b), where $n_{2}+n_{3}$ is odd, the results for fidelity are $F_{I I I E}=F_{I I E}$. 


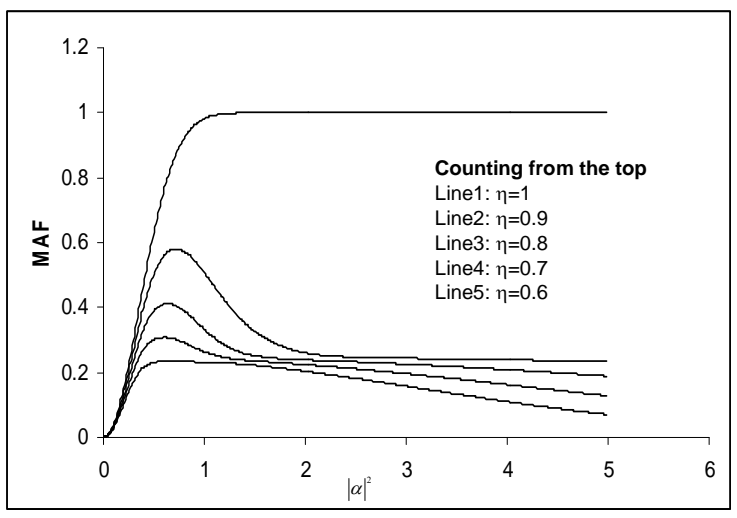

Figure 2. Figure showing variation of MAF with $|\alpha|^{2}$ for different values of $\boldsymbol{\eta}$ for non zero even counts.

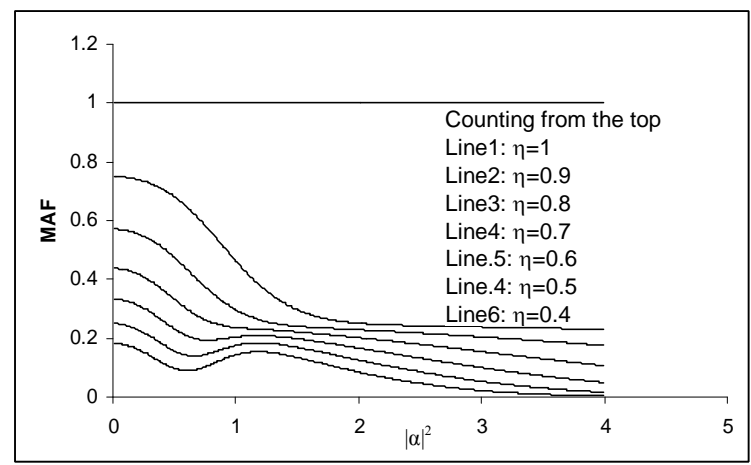

Figure 3. Figure showing variation of MAF with $|\alpha|^{2}$ for different values of $\boldsymbol{\eta}$ for odd counts.

Case IV: If Alice's measurements result in odd photons in the mode 6 and zero photon in the mode 8 , we find that the state with David is,

$$
|T\rangle \sim\left[\epsilon_{+}|-\alpha\rangle_{4}\left|-r^{\prime}\right\rangle+\epsilon_{-}|\alpha\rangle_{4}\left|r^{\prime}\right\rangle\right]
$$

For case IV (a), where $\boldsymbol{n}_{2}+\boldsymbol{n}_{3}$ is even, this is exactly the same as case II (b).

For case IV (b), where $\boldsymbol{n}_{2}+\boldsymbol{n}_{3}$ is odd, this is exactly the same as case II (a).

Case V: If Alice's measurements result in zero photon in the mode 6 and odd photon in the mode 8 , we find that the state with David is,

$$
|T\rangle \sim\left[-\epsilon_{+}|-\alpha\rangle_{4}\left|-r^{\prime}\right\rangle+\epsilon_{-}|\alpha\rangle_{4}\left|r^{\prime}\right\rangle\right]
$$

For case $\mathbf{V}(\mathbf{a})$, where $\boldsymbol{n}_{2}+\boldsymbol{n}_{3}$ is even, this is exactly the same as case (III b).

For case IV (b), where $\boldsymbol{n}_{2}+\boldsymbol{n}_{3}$ is odd, this is exactly the same as case III (a).

The variation of average fidelity

$$
F_{a v}=\sum_{i=I}^{V} P_{i} F_{i}
$$

with $|\alpha|^{2}$ for different values of $\theta$ at $\theta=0.9$ is shown in Figure 4.

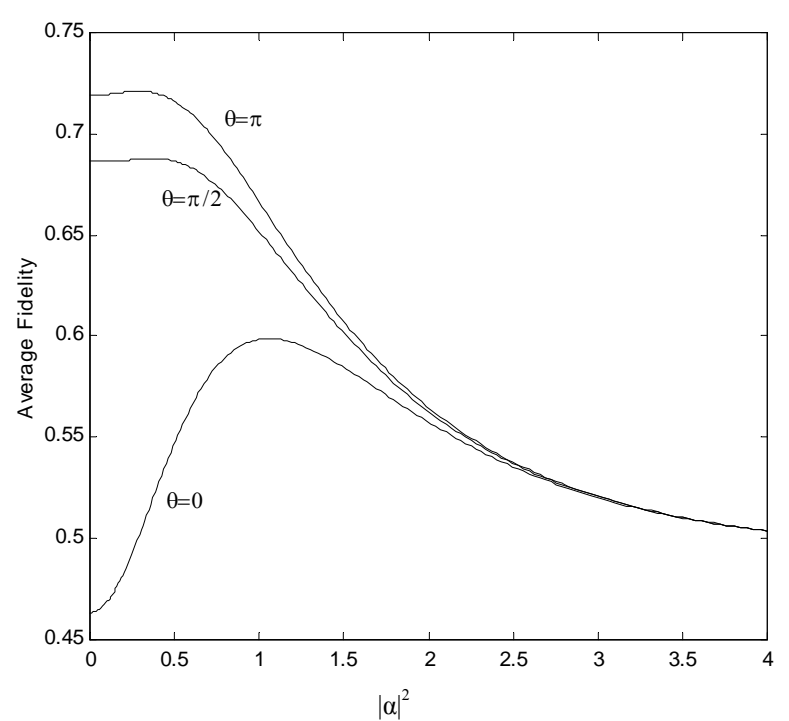

Figure 4. Figure showing variation of average fidelity with $|\alpha|^{2}$ for different values of $\boldsymbol{\theta}$ at $\boldsymbol{\eta}=\mathbf{0 . 9}$.

\section{Conclusions}

We conclude that, the scheme proposed by N. Ba An can lead to almost successful teleportation for an appreciable mean number of photons if we write $|E V E N, \alpha\rangle$ as a superposition of the vacuum state $|0\rangle$ and the non-zero even photon state $|N Z E, \alpha\rangle$, and distinguish between these results (zero and non-zero even) for even photon counts. Also, by including four parties, the security of the teleportation increases, as David cannot decipher the original information until and unless he gets the results from all Alice, Bob and Clair.

For the noiseless case we find that fidelity is unity when total photon counts $n_{6}+n_{8}+n_{2}+n_{3}$ are odd. In this case further, if $n_{8}=0$, no unitary transformation is required. For even photon counts $n_{6}+n_{8}+n_{2}+n_{3}$, a unitary transformation is required and the fidelity is close to unity for an appreciable mean number of photons. These results agree with our results for teleportation by entangled coherent state [29] with similarly modified van Enk and Hirota's scheme.

While finding the fidelity with decoherence considerations, we consider the information $|I\rangle \equiv \epsilon_{+}|\alpha\rangle+\epsilon_{-}|-\alpha\rangle$, keeping in mind our aim, viz., teleportation of this quantum information state. We investigated cases with and without noise, and calculated the minimum assured fidelity (MAF), which tells the minimum amount of fidelity which is always assured to be obtained for successful teleportation. Our results for the noisy case reduces to those for noiseless case by putting $\eta=1$.

\section{Acknowledgements}

Shivani is very grateful to Amity Institute of Applied Sciences, Amity University Noida who encouraged her 
in all aspects. Shivani is grateful to H. Prakash and N. Chandra for helpful discussions.

\section{REFERENCES}

[1] C. H. Bennett, G. Brassard, C. Crepeau, R. Jozsa, A. Peres and W. K. Wootters, "Teleporting an Unknown Quantum State via Dual Classical and Einstein-Podolsky-Rosen Channels," Physical Review Letters, Vol. 70, No. 13, 1993, pp. 1895-1899. doi:10.1103/PhysRevLett.70.1895

[2] S. J. van Enk and O. Hirota, "Entangled Coherent States: Teleportation and Decoherence," Physical Review A, Vol. 64, No. 2, 2001, Article ID: 022313. doi:10.1103/PhysRevA.64.022313

[3] X. Wang, "Quantum Teleportation of Entangled Coherent States," Physical Review A, Vol. 64, No. 2, 2001, Article ID: 022302. doi:10.1103/PhysRevA.64.022302

[4] N. Ba An, "Teleportation of Coherent-State Superpositions within a Network," Physical Review A, Vol. 68, No. 2, 2003, Article ID: 022321. doi:10.1103/PhysRevA.68.022321

[5] D. P. DiVincenzo, "Quantum Computation," Science, Vol. 270, No. 5234, 1995, pp. 255-261. doi:10.1126/science.270.5234.255

[6] C. H. Bennett and S. J. Weisner, "Communication via One- and Two-Particle Operators on Einstein-PodolskyRosen States," Physical Review Letters, Vol. 69, No. 20, 1992, pp. 2881-2884. doi:10.1103/PhysRevLett.69.2881

[7] A. K. Ekert, "Quantum Cryptography Based on Bell's Theorem," Physical Review Letters, Vol. 67, No. 6, 1991, pp. 661-663. doi:10.1103/PhysRevLett.67.661

[8] M. Murao, D. Jonathan, M. B. Plenio and V. Vedral, "Quantum Telecloning and Multiparticle Entanglement," Physical Review A, Vol. 59, No. 1, 1999, pp. 156-161. doi:10.1103/PhysRevA.59.156

[9] A. Einstein, B. Podolsky and N. Rosen, "Can QuantumMechanical Description of Physical Reality Be Considered Complete?" Physical Review, Vol. 47, No. 10, 1935, pp. 777-780. doi:10.1103/PhysRev.47.777

[10] M. K. Mishra, A. K. Maurya and H. Prakash, "Two-Way Quantum Communication: Secure Quantum Information Exchange," Journal of Physics B: Atomic, Molecular and Optical Physics, Vol. 44, 2011, Article ID: 115504.

[11] M. K Mishra and H. Prakash "Teleportation of a two Mode Entangled Coherent States," Journal of Physics B: Atomic, Molecular and Optical Physics, Vol. 43, 2010, Article ID: 185501. doi:10.1088/0953-4075/43/18/185501

[12] J.-Q. Liao and L.-M. Kuang, "Near-Complete Teleportation of Two-Mode Four-Component Entangled Coherent States," Journal of Physics B: Atomic, Molecular and Optical Physics, Vol. 40, 2007, p. 1183. doi:10.1088/0953-4075/40/6/009

[13] H. N. Phien and N. Ba An, "Quantum Teleportation of an Arbitrary Two-Mode Coherent State Using Only Linear Optics Elements," Physics Letters A, Vol. 372, No. 16, 2008, pp. 2825-2829. doi:10.1016/j.physleta.2007.12.069
[14] D. Bouwmeester, J. W. Pan, K. Mattle, M. Eibl, H. Weinfurter and A. Zeilinger, "Experimental Entanglement Swapping: Entangling Photons that Never Interacted," Nature, Vol. 390, 1997, pp. 575. doi: $10.1038 / 37539$

[15] D. Boschi, S. Branca, F. De Martini, L. Hardy and S. Popescu, "Experimental Realization of Teleporting an Unknown Pure Quantum State via Dual Classical and Einstein-Podolsky-Rosen Channels," Physical Review Letters, Vol. 80, No. 6, 1998, pp. 1121-1125. doi:10.1103/PhysRevLett.80.1121

[16] T. Ide, H. F. Hofmann, T. Kobayashi and A. Fursawa, "Continuous-Variable Teleportation of Single-Photon States via Classical Channel," Physical Review A, Vol. 66, 2002, Article ID: 011213

[17] M. Ikram, S.-Y Zhu and M. S. Zubairy, "Quantum Teleportation of an Entangled State," Physical Review A, Vol. 62, No. 2, 2000, Article ID: 022307. doi:10.1103/PhysRevA.62.022307

[18] M. S. Zubairy, "Quantum Teleportation of a Field State," Physical Review A, Vol. 58, No. 6, 1998, p. 4368. doi:10.1103/PhysRevA.58.4368

[19] A. Furusawa, J. L. Sorensen, S. L. Braunstein, C. A. Fuchs, H. J. Kimble and E. S. Polzik, "Unconditional Quantum Teleportation," Science, Vol. 282, No. 5389, 1998, pp. 706-709.

[20] S. L. Braunstein and H. J. Kimble, "Teleportation of Continuous Quantum Variables," Physical Review Letters, Vol. 80, 1998, p. 869.

[21] L. Vaidman, "Teleportation of Quantum States," Physical Review A, Vol. 49, 1994, p. 1473. doi:10.1103/PhysRevA.49.1473

[22] T. Ide, H. F. Hofmann, T. Kobayashi and A. Fursawa, "Continuous-Variable Teleportation of Single-Photon States," Physical Review A, Vol. 65, No. 1, 2001, Article ID: 012313. doi:10.1103/PhysRevA.65.012313

[23] B. C. Sanders, "Entangled Coherent States," Physical Review A, Vol. 45, No. 9, 1992, p. 6811. doi:10.1103/PhysRevA.45.6811

[24] H. Jeong, W. Son, M. S. Kim, D. Ahn and C. Brukner, "Quantum Nonlocality Test for Continuous-Variable States with Dichotomic Observables," Physical Review A, Vol. 67, No. 1, 2003, Article ID: 012106. doi:10.1103/PhysRevA.67.012106

[25] H. Jeong, M. S. Kim and J. Lee, "Quantum-Information Processing for a Coherent Superposition State via a Mixed Entangled Coherent Channel," Physical Review A, Vol. 64, No. 5, 2001, Article ID: 052308. doi:10.1103/PhysRevA.64.052308

[26] H. Jeong and M. S. Kim, "Purification of Entangled Coherent States," Quantum Information and Computation, Vol. 2, 2002, p. 208.

[27] H.-Y. Fan and H.-L. Lu An, "New Two-Mode Coherent-Entangled State and Its Application," Journal of Physics A, Vol. 37, No. 45, 2004, Article ID: 10993. doi:10.1088/0305-4470/37/45/017

[28] H. Prakash, N. Chandra, R. Prakash and Shivani, "Entanglement Diversion between Two Pairs of Entangled Co- 
herent States: Fidelity and Decoherence," International Journal of Modern Physics B, Vol. 23, No. 4, 2009, p. 585.

[29] H. Prakash, N. Chandra, R. Prakash and Shivani, "Swapping between Two Pairs of Non-Orthogonal Entangled Coherent States," International Journal of Modern Physics B, Vol. 23, No. 8, 2009, p. 2083.

[30] H. Prakash, N. Chandra, R. Prakash and Shivani, "Effect of Decoherence on Fidelity in Teleportation of Entangled Coherent States," International Journal of Quantum Information, Vol. 6, No. 5, 2008, p. 1077. doi:10.1142/S0219749908004213

[31] H. Prakash, N. Chandra, R. Prakash and Shivani, "Effect of Decoherence on Fidelity in Teleportation Using Entangled Coherent States," Journal of Physics B: Atomic, Molecular and Optical Physics, Vol. 40, No. 8, 2007, p. 1613. doi:10.1088/0953-4075/40/8/012

[32] H. Prakash, N. Chandra, R. Prakash and Shivani, "Improving the Teleportation of Entangled Coherent States," Physical Review A, Vol. 75, No. 4, 2007, Article ID:
044305. doi:10.1103/PhysRevA.75.044305

[33] H. Prakash, N. Chandra, R. Prakash and Shivani, "Improving the Entanglement Diversion between Two Pairs of Entangled Coherent States," International Journal of Modern Physics B, Vol. 24, No. 17, 2010, p. 3331.

[34] H. Prakash, N. Chandra, R. Prakash and Shivani, "Almost Perfect Teleportation Using Entangled States," International Journal of Modern Physics B, Vol. 24, No. 17, 2010, p. 3383.

[35] V. V. Dodonov, I. A. Malkin and V. I. Man'ko, "Even and Odd Coherent States and Excitations of a Singular Oscillator," Physica A, Vol. 72, No. 3, 1974, p. 597.

[36] W. K. Lai, V. Buzek and P. L. Knight, "Nonclassical Fields in a Linear Directional Coupler," Physical Review A, Vol. 43, No. 11, 1991, p. 6323. doi:10.1103/PhysRevA.43.6323

[37] Y. K. Cheong, H. Kim and H. W. Lee, "Near-Complete Teleportation of a Superposed Coherent State," Physical Review A, Vol. 70, No. 3, 2004, Article ID: 032327. doi:10.1103/PhysRevA.70.032327 


\section{Appendix A}

Case I: If Alice's measurements result in zero photons in both modes, we find that the state with David is,

$$
\begin{aligned}
\left|T^{\prime}\right\rangle & \sim\left(\epsilon_{+}+\epsilon_{-}\right)\left(|\alpha\rangle_{4}-|-\alpha\rangle_{4}\right)\left[\frac{1+x^{2}}{2}|E V E N, E V E N\rangle_{2,3}+\frac{1-x^{2}}{2}|O D D, O D D\rangle_{2,3}\right] \\
& +\left(\epsilon_{+}+\epsilon_{-}\right)\left(|\alpha\rangle_{4}+|-\alpha\rangle_{4}\right)\left[\frac{\sqrt{1-x^{4}}}{2}\left(|E V E N, O D D\rangle_{2,3}+|O D D, E V E N\rangle_{2,3}\right)\right]
\end{aligned}
$$

Case II: If Alice's measurements result in non-zero even photons in the mode 6 and zero photon in the mode 8, we find that the state with David is,

$$
\begin{aligned}
\left|T^{\prime}\right\rangle \sim\left(\epsilon_{+}|\alpha\rangle_{4}-\epsilon_{-}|-\alpha\rangle_{4}\right)\left[\frac{1+x^{2}}{2}|E V E N, E V E N\rangle_{2,3}+\frac{1-x^{2}}{2}|O D D, O D D\rangle_{2,3}\right] \\
+\left(\epsilon_{+}|\alpha\rangle_{4}+\epsilon_{-}\right)|-\alpha\rangle_{4}\left[\frac{\sqrt{1-x^{4}}}{2}\left(|E V E N, O D D\rangle_{2,3}+|O D D, E V E N\rangle_{2,3}\right)\right]
\end{aligned}
$$

Case III: If Alice's measurements result in zero photon in the mode 6 and non-zero even photons in the mode 8, we find that the state with David is,

$$
\begin{aligned}
\left.\left|T^{\prime}\right\rangle \sim\left(\epsilon_{+}|-\alpha\rangle_{4}+\epsilon_{-}|\alpha\rangle_{4}\right)\left[\frac{1+x^{2}}{2} \mid \text { EVEN, EVEN }\right\rangle_{2,3}+\frac{1-x^{2}}{2}|O D D, O D D\rangle_{2,3}\right] \\
+\left(\epsilon_{+}|-\alpha\rangle_{4}+\epsilon_{-}\right)|\alpha\rangle_{4}\left[\frac{\sqrt{1-x^{4}}}{2}\left(|E V E N, O D D\rangle_{2,3}+|O D D, E V E N\rangle_{2,3}\right)\right]
\end{aligned}
$$

Case IV: If Alice's measurements result in odd photons in the mode 6 and zero photon in the mode 8, we find that the state with David is,

$$
\begin{aligned}
\left|T^{\prime}\right\rangle \sim\left(\epsilon_{+}|\alpha\rangle_{4}+\epsilon_{-}|-\alpha\rangle_{4}\right)\left[\frac{1+x^{2}}{2}|E V E N, E V E N\rangle_{2,3}+\frac{1-x^{2}}{2}|O D D, O D D\rangle_{2,3}\right] \\
+\left(\epsilon_{+}|\alpha\rangle_{4}-\epsilon_{-}\right)|-\alpha\rangle_{4}\left[\frac{\sqrt{1-x^{4}}}{2}\left(|E V E N, O D D\rangle_{2,3}+|O D D, E V E N\rangle_{2,3}\right)\right]
\end{aligned}
$$

Case V: If Alice's measurements result in zero photon in the mode 6 and odd photon in the mode 8 , we find that the state with David is,

$$
\begin{aligned}
\left|T^{\prime}\right\rangle & \left.\sim\left(\epsilon_{+}|-\alpha\rangle_{4}+\epsilon_{-}|\alpha\rangle_{4}\right)\left[\frac{1+x^{2}}{2} \mid \text { EVEN, EVEN }\right\rangle_{2,3}+\frac{1-x^{2}}{2}|O D D, O D D\rangle_{2,3}\right] \\
& +\left(\epsilon_{+}|-\alpha\rangle_{4}-\epsilon_{-}\right)|\alpha\rangle_{4}\left[\frac{\sqrt{1-x^{4}}}{2}\left(|E V E N, O D D\rangle_{2,3}+|O D D, E V E N\rangle_{2,3}\right)\right]
\end{aligned}
$$

\section{Appendix B}

Case I: If Alice's measurements result in zero photons in both modes, we find that the state with David is,

$$
\left|T^{\prime}\right\rangle \sim\left(\epsilon_{+}|k\rangle_{R 0}+\epsilon_{-}|-k\rangle_{R 0}\right)\left[|\{\alpha\}\rangle_{234}|K\rangle_{R 1234}-|\{-\alpha\}\rangle_{234}|-K\rangle_{R 1234}\right]
$$

For Case I (a), where, $\boldsymbol{n}_{2}+\boldsymbol{n}_{3}$ is even, David's state collapses into the state,

$$
|T\rangle=\left[\epsilon_{+}\left(|\alpha\rangle_{4}|r\rangle-|-\alpha\rangle_{4}\left|-r^{\prime}\right\rangle\right)+\epsilon_{-}\left(|\alpha\rangle_{4}\left|r^{\prime}\right\rangle-|-\alpha\rangle_{4}|-r\rangle\right)\right]
$$

In terms of even and odd coherent states, the above state becomes 


$$
\begin{aligned}
|T\rangle= & \frac{1}{2 \sqrt{N}}\left\{\left[A_{+} \sqrt{\frac{1+x^{2}}{1+x^{2}}}\left(|r\rangle-|-r\rangle+\left|r^{\prime}\right\rangle-\left|-r^{\prime}\right\rangle\right)+A_{-} \sqrt{\frac{1+x^{2}}{1-x^{2}}}\left(|r\rangle+|-r\rangle-\left|r^{\prime}\right\rangle-\left|-r^{\prime}\right\rangle\right)\right]|E V E N, \alpha\rangle_{4}\right. \\
& \left.+\left[A_{+} \sqrt{\frac{1-x^{2}}{1+x^{2}}}\left(|r\rangle+|-r\rangle+\left|r^{\prime}\right\rangle+\left|-r^{\prime}\right\rangle\right)+A_{-} \sqrt{\frac{1-x^{2}}{1-x^{2}}}\left(|r\rangle-|-r\rangle-\left|r^{\prime}\right\rangle+\left|-r^{\prime}\right\rangle\right)\right]|O D D, \alpha\rangle_{4}\right\}
\end{aligned}
$$

Here,

$$
N=2\left(1-x^{2} p^{6}\right)\left[\left(1-x^{2} p^{2}\right)+\left(p^{2}-x^{2}\right)\left(\left|A_{+}\right|^{2}-\left|A_{-}\right|^{2}\right)\right]\left(1-x^{4}\right)^{-1}
$$

The fidelity $F_{I E}$ is found to be

$$
\begin{aligned}
F_{I E}= & \frac{2 x^{2}}{p\left(1-x^{4}\right)\left(1-x^{2} p^{6}\right)}\left[\left(1+x^{2} p^{8}\right)\left(\left|A_{+}\right|^{2} \cosh ^{2} \chi+\left|A_{-}\right|^{2} \sinh ^{2} \chi\right)\right. \\
& \left.-\left(x^{2}+p^{8}\right)\left(\left|A_{+}\right|^{2} \cosh ^{2} \chi-\left|A_{-}\right|^{2} \sinh ^{2} \chi\right)\right]
\end{aligned}
$$

where

$$
p=\exp \left[-(1-\eta)|\alpha|^{2}\right], \chi=|\alpha|^{2} \sqrt{\eta}
$$

This reduces to the result of Section 2, $F_{I}=\left|A_{-}\right|^{2}$, on putting $\eta=1$.

For Case I (b), where, $\boldsymbol{n}_{2}+\boldsymbol{n}_{3}$ is odd, David's state collapses into the state,

$$
|T\rangle=\left[\epsilon_{+}\left(|\alpha\rangle_{4}|r\rangle+|-\alpha\rangle_{4}\left|-r^{\prime}\right\rangle\right)+\epsilon_{-}\left(|\alpha\rangle_{4}\left|r^{\prime}\right\rangle+|-\alpha\rangle_{4}|-r\rangle\right)\right]
$$

In terms of even and odd coherent states, the above state becomes

$$
\begin{aligned}
|T\rangle= & \frac{1}{2 \sqrt{N}}\left\{\left[A_{+} \sqrt{\frac{1+x^{2}}{1+x^{2}}}\left(|r\rangle+|-r\rangle+\left|r^{\prime}\right\rangle+\left|-r^{\prime}\right\rangle\right)+A_{-} \sqrt{\frac{1+x^{2}}{1-x^{2}}}\left(|r\rangle-|-r\rangle-\left|r^{\prime}\right\rangle+\left|-r^{\prime}\right\rangle\right)\right]|E V E N, \alpha\rangle_{4}\right. \\
& \left.+\left[A_{+} \sqrt{\frac{1-x^{2}}{1+x^{2}}}\left(|r\rangle-|-r\rangle+\left|r^{\prime}\right\rangle-\left|-r^{\prime}\right\rangle\right)+A_{-} \sqrt{\frac{1-x^{2}}{1-x^{2}}}\left(|r\rangle+|-r\rangle-\left|r^{\prime}\right\rangle-\left|-r^{\prime}\right\rangle\right)\right]|O D D, \alpha\rangle_{4}\right\}
\end{aligned}
$$

Here,

$$
N=2\left(1+x^{2} p^{6}\right)\left[\left(1-x^{2} p^{2}\right)+\left(p^{2}-x^{2}\right)\left(\left|A_{+}\right|^{2}-\left|A_{-}\right|^{2}\right)\right]\left(1-x^{4}\right)^{-1}
$$

The fidelity $F_{I O}$ is found to be

$$
\begin{aligned}
F_{I O}= & \frac{2 x^{2}}{p\left(1-x^{4}\right)\left(1+x^{2} p^{6}\right)}\left[\left(1-x^{2} p^{8}\right)\left(\left|A_{+}\right|^{2} \cosh ^{2} \chi+\left|A_{-}\right|^{2} \sinh ^{2} \chi\right)\right. \\
& \left.-\left(x^{2}-p^{8}\right)\left(\left|A_{+}\right|^{2} \cosh ^{2} \chi-\left|A_{-}\right|^{2} \sinh ^{2} \chi\right)\right]
\end{aligned}
$$

This reduces to the result of Section 2, $F_{I O}=\left|A_{+}\right|^{2}$, on putting $\eta=1$.

Case II: If Alice's measurements result in non-zero even photons in the mode 6 and zero photon in the mode 8 , we find that the state with David is,

$$
\left|T^{\prime}\right\rangle \sim\left[\epsilon_{+}|\{\alpha\}\rangle_{234}|r\rangle-\epsilon_{-}|\{-\alpha\}\rangle_{234}|-r\rangle\right]
$$

For case II (a), where $\boldsymbol{n}_{2}+\boldsymbol{n}_{3}$ is even, David's state collapses into the state,

$$
|T\rangle \sim\left[\epsilon_{+}|\alpha\rangle_{4}|r\rangle-\epsilon_{-}|-\alpha\rangle_{4}|-r\rangle\right]
$$

In terms of even and odd coherent states, the above state becomes 


$$
\begin{aligned}
\left|T^{\prime}\right\rangle= & \frac{1}{2 \sqrt{N}}\left\{\left[A_{+} \sqrt{\frac{1+x^{2}}{1+x^{2}}}(|r\rangle-|-r\rangle)+A_{-} \sqrt{\frac{1+x^{2}}{1-x^{2}}}(|r\rangle+|-r\rangle)\right] \mid \text { EVEN, } \alpha\right\rangle_{4} \\
& \left.+\left[A_{+} \sqrt{\frac{1-x^{2}}{1+x^{2}}}(|r\rangle+|-r\rangle)+A_{-} \sqrt{\frac{1-x^{2}}{1-x^{2}}}(|r\rangle-|-r\rangle)\right]|O D D, \alpha\rangle_{4}\right\}
\end{aligned}
$$

David may apply the unitary transformation

$$
U=|E V E N, \alpha\rangle_{4}{ }_{4}\langle O D D, \alpha|+| O D D, \alpha\rangle_{4}{ }_{4}\langle E V E N, \alpha|
$$

found in Section 2 and get the state

$$
\begin{aligned}
\left|T^{\prime}\right\rangle= & \frac{1}{2 \sqrt{N}}\left\{\left[A_{+} \sqrt{\frac{1+x^{2}}{1+x^{2}}}(|r\rangle+|-r\rangle)+A_{-} \sqrt{\frac{1+x^{2}}{1-x^{2}}}(|r\rangle-|-r\rangle)\right]|E V E N, \alpha\rangle_{4}\right. \\
& \left.+\left[A_{+} \sqrt{\frac{1-x^{2}}{1+x^{2}}}(|r\rangle-|-r\rangle)+A_{-} \sqrt{\frac{1-x^{2}}{1-x^{2}}}(|r\rangle+|-r\rangle)\right]|O D D, \alpha\rangle_{4}\right\}
\end{aligned}
$$

Here,

$$
N=\left[2\left(1+x^{4} p^{8}\right)-x^{2}\left(1-p^{8}\right)\right]\left[\left(\left|A_{+}\right|^{2}-\left|A_{-}\right|^{2}\right)\right]\left(1-x^{4}\right)^{-1}
$$

The fidelity $F_{I I E}$ for this case is found to be

$$
\begin{aligned}
F_{I I E}= & Z\left\{\left[\left(X \mathrm{e}^{\chi}-Y \mathrm{e}^{-\chi}\right)+\left(X \mathrm{e}^{-\chi}-Y \mathrm{e}^{\chi}\right)\left(\left|A_{+}\right|^{2}-\left|A_{-}\right|^{2}\right)\right]^{2}\left(1+p^{10}\right)\right. \\
& +4\left[\left|A_{+}\right|^{2}\left|A_{-}\right|^{2}\left[\left(1+x^{4}\right)\left(\cosh ^{2} \chi+\sinh ^{2} \chi\right)-2 x^{2}\right]\right. \\
& \left.\left.+\left(1-x^{4}\right)\left(A_{+}^{2} A_{-}^{* 2}+A_{-}^{2} A_{+}^{* 2}\right) \cosh \chi \sinh \chi\right]\left(1-p^{10}\right)\left(1-x^{4}\right)\right\}
\end{aligned}
$$

where

$$
X=1+x^{2} x^{2} ; Y=x^{2}+x^{2} \text { and } Z=2^{-1} x^{2} p^{-1} N^{-1}\left(1-x^{4}\right)^{-2}\left(1-x^{4}\right)^{-1}
$$

This reduces to equation (V.22) for $\eta=1$ (the noiseless case).

For case II (b), where $\boldsymbol{n}_{2}+\boldsymbol{n}_{3}$ is even, David's state collapses into the state,

$$
|T\rangle=\left[\epsilon_{+}|\alpha\rangle_{4}|r\rangle+\epsilon_{-}|-\alpha\rangle_{4}|-r\rangle\right]
$$

In terms of even and odd coherent states, the above state becomes

$$
\begin{aligned}
|T\rangle= & \frac{1}{2 \sqrt{N}}\left\{\left[A_{+} \sqrt{\frac{1+x^{2}}{1+x^{2}}}(|r\rangle+|-r\rangle)+A_{-} \sqrt{\frac{1+x^{2}}{1-x^{2}}}(|r\rangle-|-r\rangle)\right] \mid \text { EVEN, } \alpha\right\rangle_{4} \\
& \left.+\left[A_{+} \sqrt{\frac{1-x^{2}}{1+x^{2}}}(|r\rangle-|-r\rangle)+A_{-} \sqrt{\frac{1-x^{2}}{1-x^{2}}}(|r\rangle+|-r\rangle)\right]|O D D, \alpha\rangle_{4}\right\}
\end{aligned}
$$

where

$$
N=\left[2\left(1-x^{4} p^{8}\right)-x^{2}\left(1-p^{8}\right)\right]\left[\left(\left|A_{+}\right|^{2}-\left|A_{-}\right|^{2}\right)\right]\left(1-x^{4}\right)^{-1}
$$

No unitary transformation is required in this case.

The fidelity $F_{I I O}$ for this case is calculated to be, 


$$
\begin{aligned}
F_{I I O}= & \frac{2 x^{2}\left(1-x^{4}\right)}{p\left\{\left(1-x^{4} p^{8}\right)-x^{2}\left(1-p^{8}\right)\left(\left|A_{+}\right|^{2}-\left|A_{-}\right|^{2}\right)\right\}} \times\left\{\left[\frac{\left|A_{+}\right|^{2} \cosh \chi}{\left(1+x^{2}\right)}+\frac{\left|A_{-}\right|^{2} \sinh \chi}{\left(1-x^{2}\right)}\right]^{2}\left(1+p^{10}\right)\right. \\
& \left.+\left[\left|A_{+}\right|^{2}\left|A_{-}\right|^{2}\left(\cosh ^{2} \chi+\sinh ^{2} \chi\right)+\left(A_{+}^{2} A_{-}^{* 2}+A_{-}^{2} A_{+}^{* 2}\right) \cosh \chi \sinh \chi\right] \frac{\left(1-p^{10}\right)}{\left(1-x^{4}\right)}\right\}
\end{aligned}
$$

It is seen that $F_{I I O}=1$ for the noiseless case $\eta=1$.

Case III: If Alice's measurements result in zero photon in the mode 6 and non-zero even photons in the mode 8 , we find that the state with David is,

$$
\left|T^{\prime}\right\rangle \sim\left[-\epsilon_{+}|\{-\alpha\}\rangle_{234}\left|-r^{\prime}\right\rangle+\epsilon_{-}|\{\alpha\}\rangle_{234}\left|r^{\prime}\right\rangle\right]
$$

For case III (a), where $\boldsymbol{n}_{2}+\boldsymbol{n}_{3}$ is even, David's state collapses into the state,

$$
|T\rangle \sim\left[-\epsilon_{+}|-\alpha\rangle_{4}\left|-r^{\prime}\right\rangle+\epsilon_{-}|\alpha\rangle_{4}\left|r^{\prime}\right\rangle\right]
$$

In terms of even and odd coherent states, the above state becomes

$$
\begin{aligned}
\left|T^{\prime}\right\rangle= & \frac{1}{2 \sqrt{N}}\left\{\left[A_{+} \sqrt{\frac{1+x^{2}}{1+x^{2}}}\left(\left|r^{\prime}\right\rangle-\left|-r^{\prime}\right\rangle\right)+A_{-} \sqrt{\frac{1+x^{2}}{1-x^{2}}}\left(\left|r^{\prime}\right\rangle+\left|-r^{\prime}\right\rangle\right)\right]|E V E N, \alpha\rangle_{4}\right. \\
& \left.+\left[A_{+} \sqrt{\frac{1-x^{2}}{1+x^{2}}}\left(\left|r^{\prime}\right\rangle+\left|-r^{\prime}\right\rangle\right)+A_{-} \sqrt{\frac{1-x^{2}}{1-x^{2}}}\left(\left|r^{\prime}\right\rangle-\left|-r^{\prime}\right\rangle\right)\right]|O D D, \alpha\rangle_{4}\right\}
\end{aligned}
$$

The required unitary transformation for this case is,

$$
U=|E V E N, \alpha\rangle_{44}\langle O D D, \alpha|-| O D D, \alpha\rangle_{44}\langle E V E N, \alpha|
$$

This leads to the teleported state,

$$
\begin{aligned}
\left|T^{\prime}\right\rangle= & \frac{1}{2 \sqrt{N}}\left\{\left[A_{+} \sqrt{\frac{1+x^{2}}{1+x^{2}}}\left(\left|r^{\prime}\right\rangle+\left|-r^{\prime}\right\rangle\right)+A_{-} \sqrt{\frac{1+x^{2}}{1-x^{2}}}\left(\left|r^{\prime}\right\rangle-\left|-r^{\prime}\right\rangle\right)\right]|E V E N, \alpha\rangle_{4}\right. \\
& \left.+\left[A_{+} \sqrt{\frac{1-x^{2}}{1+x^{2}}}\left(\left|r^{\prime}\right\rangle-\left|-r^{\prime}\right\rangle\right)+A_{-} \sqrt{\frac{1-x^{2}}{1-x^{2}}}\left(\left|r^{\prime}\right\rangle+\left|-r^{\prime}\right\rangle\right)\right]|O D D, \alpha\rangle_{4}\right\}
\end{aligned}
$$

For case III (b), where $\boldsymbol{n}_{2}+\boldsymbol{n}_{3}$ is odd, David's state collapses into the state,

$$
|T\rangle \sim\left[\epsilon_{+}|-\alpha\rangle_{4}\left|-r^{\prime}\right\rangle+\epsilon_{-}|\alpha\rangle_{4}\left|r^{\prime}\right\rangle\right]
$$

In terms of even and odd coherent states, the above state becomes

$$
\begin{aligned}
\left|T^{\prime}\right\rangle= & \frac{1}{2 \sqrt{N}}\left\{\left[A_{+} \sqrt{\frac{1+x^{2}}{1+x^{2}}}\left(\left|r^{\prime}\right\rangle+\left|-r^{\prime}\right\rangle\right)-A_{-} \sqrt{\frac{1+x^{2}}{1-x^{2}}}\left(\left|r^{\prime}\right\rangle-\left|-r^{\prime}\right\rangle\right)\right]|E V E N, \alpha\rangle_{4}\right. \\
& \left.+\left[A_{+} \sqrt{\frac{1-x^{2}}{1+x^{2}}}\left(\left|r^{\prime}\right\rangle-\left|-r^{\prime}\right\rangle\right)-A_{-} \sqrt{\frac{1-x^{2}}{1-x^{2}}}\left(\left|r^{\prime}\right\rangle+\left|-r^{\prime}\right\rangle\right)\right]|O D D, \alpha\rangle_{4}\right\}
\end{aligned}
$$

For this case required unitary transformation is

$$
U=|E V E N, \alpha\rangle_{44}\langle E V E N, \alpha|-| O D D, \alpha\rangle_{44}\langle O D D, \alpha|
$$

The teleported state becomes 


$$
\begin{aligned}
|T\rangle= & \frac{1}{2 \sqrt{N}}\left\{\left[A_{+} \sqrt{\frac{1+x^{2}}{1+x^{2}}}\left(\left|r^{\prime}\right\rangle+\left|-r^{\prime}\right\rangle\right)-A_{-} \sqrt{\frac{1+x^{2}}{1-x^{2}}}\left(\left|r^{\prime}\right\rangle-\left|-r^{\prime}\right\rangle\right)\right]|E V E N, \alpha\rangle_{4}\right. \\
& \left.+\left[-A_{+} \sqrt{\frac{1-x^{2}}{1+x^{2}}}\left(\left|r^{\prime}\right\rangle-\left|-r^{\prime}\right\rangle\right)+A_{-} \sqrt{\frac{1-x^{2}}{1-x^{2}}}\left(\left|r^{\prime}\right\rangle+\left|-r^{\prime}\right\rangle\right)\right]|O D D, \alpha\rangle_{4}\right\}
\end{aligned}
$$

Case IV: If Alice's measurements result in odd photons in the mode 6 and zero photon in the mode 8 , we find that the state with David is,

$$
\left|T^{\prime}\right\rangle \sim\left[\epsilon_{+}|\{\alpha\}\rangle_{234}|r\rangle+\epsilon_{-}|\{-\alpha\}\rangle_{234}|-r\rangle\right]
$$

For case IV (a), where $\boldsymbol{n}_{2}+\boldsymbol{n}_{3}$ is even, David's state collapses into the state,

$$
|T\rangle \sim\left[\epsilon_{+}|\alpha\rangle_{4}|r\rangle+\epsilon_{-}|-\alpha\rangle_{4}|-r\rangle\right]
$$

For case IV (b), where $\boldsymbol{n}_{2}+\boldsymbol{n}_{3}$ is odd, David's state collapses into the state,

$$
|T\rangle \sim\left[\epsilon_{+}|\alpha\rangle_{4}|r\rangle-\epsilon_{-}|-\alpha\rangle_{4}|-r\rangle\right]
$$

Case V: If Alice's measurements result in zero photon in the mode 6 and odd photon in the mode 8, we find that the state with David is,

$$
\left|T^{\prime}\right\rangle \sim\left[\epsilon_{+}|\{-\alpha\}\rangle_{234}\left|-r^{\prime}\right\rangle+\epsilon_{-}|\{\alpha\}\rangle_{234}\left|r^{\prime}\right\rangle\right]
$$

For case V (a), where $\boldsymbol{n}_{2}+\boldsymbol{n}_{3}$ is even, David's state collapses into the state,

$$
|T\rangle \sim\left[\epsilon_{+}|-\alpha\rangle_{4}\left|-r^{\prime}\right\rangle+\epsilon_{-}|\alpha\rangle_{4}\left|r^{\prime}\right\rangle\right]
$$

For case V (b), where $\boldsymbol{n}_{2}+\boldsymbol{n}_{3}$ is odd, David's state collapses into the state,

$$
|T\rangle \sim\left[-\epsilon_{+}|-\alpha\rangle_{4}\left|-r^{\prime}\right\rangle+\epsilon_{-}|\alpha\rangle_{4}\left|r^{\prime}\right\rangle\right]
$$

